\title{
L'art comme lieu du sens et de la vérité corporelle chez Henry Bauchau
}

Le Présent d'incertitude $\mathbf{2 1 7}$, journal couvrant les années 2002-2005, est indubitablement un travail d'écriture en pratique qui accompagne la lente et finale parturition de L'Enfant bleu. Or on sait combien, chez Henry Bauchau, l'écriture des Journaux croise celle de l'œuvre et est un réel effort, puisque l'auteur confie dans Le Présent d'incertitude, le 7 mai 2002: "Retravaillé ces jours-ci à la mise au point du Passage de la Bonne-Graine. Cela aura été finalement un grand travail, comme mes deux Journaux précédents." Aussi, chaque aboutissement ou accomplissement du travail donne lieu à cette puissante tension avec le corps écrivant, qui se dit ou se rappelle dans des symptomatologies qui manifestent l'effort et les effets du cheminement ou de la remontée de l'écriture qui vient à son terme: "Le 4 décembre j'ai encore pu travailler normalement et écrire à nouveau quatre pages du roman. Je ressentais à nouveau joie et confiance. Malheureusement dès le lendemain la fièvre est revenue. Quelle résistance provoque en moi l'achèvement de ce roman que j'espère de toutes mes forces. „18

C'est une évidence, Henry Bauchau vit, souffre et jouit l'écriture, qui reste une pratique spécifique et qui n'est pas la théorisation de ce que nos métiers de lecture ou d'exégète en font, sauf si la vie théorique est une pratique radicale. Il me plaît ainsi de rappeler ce propos tenu par Henry Bauchau quant à son "propre" travail, dans son extrême vérité et son exigeant dénuement: "Visite de Régis Lefort, qui m’apporte le texte de la thèse qu'il a consacrée à L'Originel dans l'œuvre d'Henry Bauchau. Il a obtenu la mention "très honorable avec félicitations du jury". Son travail est certainement excellent, mais peut-il se douter à quel point le mien est basé sur des intuitions simples, l'écoute intérieure et les messages de la langue que j'utilise à travers l'attention aux réactions du corps? ”219 On le voit, le rapport

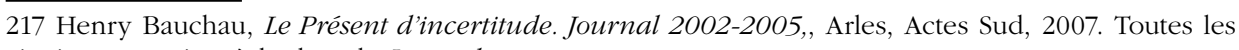
citations renvoient à la date du Journal.

2189 novembre 2002.

$2191^{\text {er }}$ février 2004. Il conviendrait de mettre également cette affirmation en perspective: "Anne Neuschafer [...] m'a posé beaucoup de questions sur mon passé auxquelles j’ai répondu aussi précisément que j'ai pu, tout en pensant qu'une œuvre de romans et de Journaux ne rapproche pas tant le lecteur de la vie au jour le jour de l'écrivain que de sa vie imaginaire, je veux dire de ce qu'il éprouve dans les moments où il n'est ni dans l'action ni dans la pensée. Nos écrits reflètent plus les trous de 
entre perspective critique et littérature ne devrait se penser que sur le plan de la vie, voire plus particulièrement sur le plan originaire et porteur d'égalisation de ce que nous appellerons, dans notre langage philosophique, le pathos de la vie, et non plus uniquement sur le plan d'une articulation conceptuelle, notionnelle, voire épistémologique, entre l'un et l'autre.

En ce sens, la littérature, comme création, dit et parle la vie avec l'imaginaire qui la caractérise, tandis que la critique, comme analyse, trouve sa part de nouveauté dans le concept, dans la pensée notionnelle, plus particulièrement. Or la vie est infiniment plus difficile à dire - ou décrire - notionnellement ou spéculativement que littérairement, si bien que le travail de la littérature - déjà entendue comme un art, et spécifiquement un art de vivre - offre des potentialités particulières du dire de l'affectivité et de l'épreuve de cette vie, avec ses tonalités, ses sonorités, ses visions, ses impressions, ses sensations, ses affects, ses pouvoirs, ses expressions, dans la mesure où la vie est un processus qui poursuit un accomplissement, en traversant l'épaisseur de la chair, pour aller vers sa propre libération.

À cet égard, je fais l'hypothèse que, dans Le Présent d'incertitude, Henry Bauchau évoque la question de l'art dans la vie sous un double prisme: son travail propre (ou de "mienneté") confronté aux écritures en cours (la prose du Journal qui se déroule chronologiquement, la création poétique afférente, sans oublier la puissance de la vie onirique) et celui se faisant d'Orion, le roman en acte, apparaissant ici ou là dans le Journal; Orion, cette figure certes "historique", mais combien prototypique de ceux qui entrent dans un travail de "passivité" et "d'obéissance" envers la "matière" de l'art, dont nous allons voir comment Henry Bauchau la détermine, pour aller ainsi vers la plus fine intériorité du moi, celle de la vie et en particulier la vie de l'Inconscient qui ne cesse de travailler, et ceci à travers des expériences esthétiques ou artistiques d'essencification et d'épreuve de cette vie.

Cependant, avant de tenter de comprendre ce que le mot "vie" entend signifier chez Henry Bauchau, rappelons, en guise de préalable, que ce "rapport conscient-inconscient" est, comme Henry Bauchau le confie le 26 avril 2003, toujours lu dans la perspective du rapport Dionysos-Apollon chez Nietzsche. Dionysos est le contre-jour de la lumière, de l'image et des formes lumineuses d'Apollon, il est ce dieu sans monde, celui du désir, celui de "la vie écrasée contre elle-même, dans sa joie et dans sa souffrance": "[...] c'est un dieu qui est chargé de soi dans un pathos si lourd qu'en effet

nos vies que les moments voulus ou médités. Ils alternent nos instants d'intensité et ceux où nous nous perdons de vue dans nos personnages ou dans nos perceptions imaginaires vécues elles aussi mais dans le "monde abîme" que nous traversons si souvent sans le percevoir en suivant les chemins, les routes et surtout les autoroutes de l'habitude. Nos vies sont sans doute beaucoup plus menées par l'inconscient et les grandes forces lentes qui régissent nos mouvements collectifs que nous ne pouvons le voir." $1^{\text {er }}$ février 2003. 
il veut se décharger de soi. Au fond, Dionysos est celui qui génère Apollon pour se mettre à l'écart de soi. „220 Dans cette perspective, Le Présent d'incertitude dit bien cette frénésie de la vie, qui est un accroissement incessant de soi, cette volonté de puissance du "ja sagen", qui pousse la vie à vouloir davantage, à voir et à éprouver, toujours et encore plus, selon un mouvement d'intensification et d'accroissement.

Là, dans la vie, tout est "de la même étoffe", entre souffrir et jouir, joie et tristesse, ivresse et désespoir, charge et décharge, au gré d'une transformation et d'une possible "modification intérieure", qui fait que, notamment dans le cas de fonctions de la création esthétique, en tant qu'expression des modalités pathétiques de la vie, le pathos de la souffrance peut se transmuer en pathos de la jouissance, puisque le sol originaire est similairement constitutif. C'est ce qu'a bien vu Nancy Huston dans la lettre rapportée par Henry Bauchau, à l'occasion de la lecture de L'Enfant bleu: "Françoise Nyssen m'a donné votre Enfant bleu à Arles, la semaine dernière et je l'ai lu dans un état "second", presque toujours au bord des larmes et le ventre noué, m'émerveillant que vous ayez su capter avec tant de précision le lien entre la souffrance et l'art, l'horreur et le geste de création. Véronique Vasco est un grand personnage aux côtés du jeune Orion - sachant, elle la nonartiste, ce qu'est l'art: mieux parfois que les artistes eux-mêmes... sachant se mettre en danger pour le faire advenir, chez son mari comme chez son patient. / C'est vous qui avez été patient, de façon magnifique (et douloureuse je le sais), ces cinq dernières années, pour que cette histoire puisse se déployer dans toute sa complexité et toute sa vérité. Vous nous faites voir, miraculeusement, ces labyrinthes et ces îles du paradis, ces monstres terrifiants et cette boue qui respire et qui chante. [...]”21

Mais alors qu'est-ce que la vie pour Henry Bauchau? Cette question est d'une très grande complexité et demanderait bien des développements. Au regard du corpus qui nous retient, nous pourrions répondre en évoquant cette affirmation: "Est-ce que l'espérance ne trompe pas, comme l'assure saint Paul? Souvent l'espérance se trompe car nous lui assignons des buts, des objectifs souvent vains, décevants, inatteignables. Mais elle ne nous trompe pas, elle est le souffle, le sang, la vie elle-même. Quand notre vie humaine s'arrête, $l a$ vie s'arrête-t-elle ou prend-elle une autre forme comme je l'espère? „222 Cette affirmation me semble être en consonance avec cette thèse phénoménologique de Michel Henry, qui écrit dans son langage spécifique: "Je pense que c'est dans la vie que naissent les individus et c'est pour cela qu'ils sont dans une situation de passivité - même si cette

220 Michel Henry, "Art et phénoménologie de la vie", dans Phénoménologie de la vie. Tome III. De l'art et du politique, Paris, Puf, 2004, p. 287.

22121 juin 2004.

22228 juin 2003. Nous soulignons. 
passivité leur donne l'occasion d'être eux-mêmes et donc d'être libres en ce sens. Pour moi les êtres humains sont beaucoup plus comme des nageurs lâchés dans un océan, supportés par lui, par ses vagues. Et c'est cela la vie: une vague qui se sent elle-même. „223

Il est cependant évident que la vie et l'art qui l'accompagne ne sont pas sans rapport avec ce qu'il faut appeler, chez Henry Bauchau, la nature ou le monde, même si, affirme Henry Bauchau, "on peut consacrer sa vie à l'art, l'art et la vie demeurent deux mondes communicants mais distincts " ${ }^{224}$. Ainsi, on mesure, d'une part, la force de cette nature, en particulier la belle nature, celles des "admirables cerisiers blancs, très hauts, tout en fleur" et qui sont "comme des apparitions d'un autre monde, plus heureux, plus pacifié, plus charnel [...]”25 que celui que vit Henry Bauchau. C'est de la sorte que la nature vit ses saisons, dont le printemps est le plus souvent le signe d'une "allégresse", d'une "renaissance", voire d'une "résurrection". C'est dire que la nature ${ }^{226}$ porte une essence propre et que la terre, en particulier, laisse exhaler des sonorités, des tonalités, des visions, qui sont l'expression de son action, la célébration, si bien que l'on peut assurément parler d'une énergie naturelle qui émane de la terre. C'est en tout cas ce que Henry Bauchau dit avoir écrit à Myriam Watthee-Delmotte quand il lui fait valoir que, en processus de création, "l'image divine [...] peut être de pierre, d'écriture ou de musique. / Ce n'est pas une fiction c'est une célébration de ce qui est, elle ne peut se faire sans reconnaître et célébrer ce qui a été et espérer ce qui sera "227.

En réalité, Henry Bauchau semble éprouver une forme de "mystique océane" ou "sauvage", voire une sorte de naturalisme spirituel ou vital, mais constamment intériorisé. On en prendra pour preuve cette narration d'un séjour prolongé à l'hôpital, en février 2005, dans "ce lieu collectif, efficient, technicisé" où l'homme éprouve un "manque de parole" qui le réduit à n'être que "le symptôme pour lequel [il est] là". Mais il ajoute également: "Pendant ces jours, temps brouillé, grand froid, neige, soleil admirable. Tout cela à l'extérieur de ma vie enfermée. La matière agit, est agie,

223 Michel Henry, "Narrer le pathos", dans Phénoménologie de la vie. Tome III. De l'art et du politique, op. cit., p. 321.

22427 avril 2003

2253 avril 2002. Nous soulignons.

226 La contemplation de la nature, en particulier celle des arbres, confère à Henry Bauchau la force et le bonheur: "Bonheur des grands troncs qui s'élèvent et dont je reçois la force dans mon torse fatigué, mes muscles presque disparus. La terre chante ces jours-ci, tout éclairée de bleus, de verts. Elle ne chante pas de bonheur, elle chante d'exister. Elle célèbre l'existence de l'existence." 3 avril 2002. Et on pourrait ajouter à cet optimisme naturaliste la contemplation des oiseaux, au sujet de laquelle Henry Bauchau note: "Les hirondelles/ boivent en vol / C'est Conrad Stein qui m'a dit cela un matin. C'est un fait d'un autre monde, où la pesanteur semble moins compter que dans le nôtre où la grâce est rare et lourdeur et maladresse habituelles." 22 juillet 2002.

2279 mai 2005. 
se transforme, je devine cette puissante résurrection, cette forte résistance aussi de sa gigantesque matière. Changer de saison, d'esprit ou d'âme ne va pas sans efforts. Au moins ai-je appris cela. „228

Cependant, cette "mystique océane" n'est sur aucun point une mystique de la fusion, dans la mesure où elle instaure le paradigme de la différence, dont nous verrons plus loin toute la force pertinente pour penser et vivre la création artistique, dans le temps et l'espace. On peut déjà toutefois en dire que le mot en sera l'expression, en raison de sa capacité médiatrice, hors espace et hors temps, si l'on en croit cette réflexion sur la tension entre prose et poésie. Henry Bauchau y confie l'exigence du "travail" qui épuise et est "un travail d'attente, de recherche de l'identification du mot à ce qu'[il] per[çoit] en [lui] de la réalité du jardin, de sa profondeur de vie, de ses couleurs, de ses ciels, de la maison de bois d'un autre siècle, des sons qui traversaient l'espace enchanté par instants de [s]on corps "229.

On peut également en dire que le corps sera également le lieu de tension, tant dans sa corporéité concrète et temporelle que dans sa portée la plus spirituelle, celle dont Henry Bauchau aime à dire qu'elle est marquée par le "tumulte" des dieux en lui. Des "dieux-pulsions qui saluent longtemps en nous le désir, le plaisir, la séduction" et qui, l'âge venant, "nous tournent vers la contemplation des choses, vers l'identification à ce qui est ${ }^{230}$, ce "ce qui est" pouvant être un élément naturel, comme les fameux cèdres du Liban et le prunus blanc du Jardin des plantes, qui sont véritablement des "lieux d'identification "231. On peut enfin en dire que le travail, au sens précis conféré à ce mot, en sera le principe de différence, par exemple. Une ample citation du Journal mérite d'être faite à cet égard: "Il est juste que notre désir d'anéantissement dans l'inconnaissable ou en Dieu soit freiné car c'est un désir de mort prématurée, de paix totale, contraire à la voie de l'amour qui est pleine de doute et de turbulence comme la vie. / Il est bon aussi que notre désir d'accéder au Tout et de nous fondre en lui soit également freiné. Tant que nous sommes une partie du Tout, la vérité est de nous comporter en partie, il y a de la présomption à vouloir atteindre le Tout. La vérité est de prosterner notre esprit et notre corps devant le rien et le tout, de reconnaître

22826 février 2005. On peut également mettre en perspective le propos avec cette affirmation: "Marc [Quaghebeur] me dit que nous sommes au bord d'une révolution fondamentale, qui sera moins l'œuvre des hommes qui, pour le moment, désirent surtout consommer librement, mais qui naîtra des réactions de la Terre à nos actes inconsidérés et à l'épuisement des ressources actuelles d'énergie qui risquent de provoquer des catastrophes. Je partage ce point de vue et crois comme lui qu'il n'y a pas pour le moment de mobiles suffisants pour amener les gens à changer, à renoncer à la civilisation du pétrole, de l'automobile et du déchet: nous allons vers la catastrophe et si nous réagissons ce sera trop tard. Comme je sens la présence de la mort dans mon corps depuis cet hiver, je sens la catastrophe écologique inscrite dans l'avenir. " 27 mai 2005.

22923 octobre 2004. Nous soulignons.

2306 décembre 2004

231 Idem. 
qu'ils sont inatteignables pour nous sauf risque d'éclatement dans l'orgueil ou la folie. Il faut regarder autant que possible dans ces deux directions mais le frein en nous est bienfaisant et nécessaire. Surtout si c'est le frein du travail qui ne permet pas les grands abandons au Rien, ni au Tout. „232

Il est ainsi patent qu'il est une mise en garde devant l'hybris égotique, qui va véritablement de pair avec les exigences du "travail", exprimant ainsi quelque chose de la spécificité de la création artistique, dans la mesure où elle n'est point "œuvre", puisque c'est bien la dynamique de la vie qu'il convient de laisser advenir, mieux "l'être intérieur", celui dont Henry Bauchau, conforté par Pierre Jean Jouve, dit qu'il ne "ne se développe pas par l'œuvre faite, mais par le travail de ce qui est encore à faire „233. Mais pourquoi en est-il de la sorte? C'est sur ce point qu'une brève évocation des rapports entre l'art et la nature mérite d'être avancée. En citant son ami Adriano Marchetti, Henry Bauchau note: "La physis ne dit rien, elle fait signe, il appartient à l'homme de décider. Mais cette décision est un destin, parce que l'bybris est rendue possible par le pouvoir même de la matière (energeia), dont nous faisons partie et dont nous nous emparons. „234

Or, en ce sens, c'est qu'il est bien une part de sacré dans la nature que l'homme doit vénérer ou devant lequel il doit se recueillir ("relegere»), part à laquelle Henry Bauchau confère le nom d'“inespéré" et d'indéchiffré „235. Il l'explique de la sorte: "L'indéchiffré, c'est ce que j'ai ressenti après mes longues contemplations des chardons bleus, du jardin au soleil levant et du hêtre rouge. Il y a dans les plantes comme dans les choses une part, non pas indéchiffrable, on peut comprendre, on peut expliquer, mais qui demeure indéchiffrée. C'est la part du fondamentalement autre, qui vit de sa propre vie, non de la nôtre et des constructions du savoir. "236 C'est donc que chaque homme reçoit ce devoir de "déchiffrer" sa vie, c'est-à-dire cette "tâche très mystérieuse qui [...] demande de surmonter bien des résistances et des échecs, de perdre nos forces, d'accepter nos défaillances de mémoire et de lucidité „237, quelle que soit la saison de l'âge ou de l'année.

S'il en est ainsi, c'est que l'art - en ses diverses activités - est en immédiate relation de constitution avec ces forces et formes de la nature, mais aussi la matière. Il est, en effet, frappant de voir combien Le Présent d'incertitude est sous-tendu par une véritable méditation de Simone Weil, au sujet de laquelle Henry Bauchau écrit le 20 avril 2002, en la citant: "La matière est entière passivité et par suite entière obéissance à la volonté de Dieu. Elle

2329 juin 2002.

2336 février 2005.

2345 décembre 2002.

23531 juillet 2004

$2361^{\text {er }}$ août 2004 . Nous soulignons.

23724 décembre 2004. 
est pour nous un parfait modèle" / "Par la joie la beauté du monde pénètre dans notre âme. Par la douleur elle nous entre dans le corps" "Puis, il ajoute presque aussitôt: "Je relis Simone Weil, elle me touche autant qu'au début des années 1970. Elle a écrit dans une période historique d'extrême malheur qui retentit sur sa vision. Nous sommes plus prospères, mais le monde n'a pas vraiment changé. Les divers appareils qui, depuis son temps, facilitent la communication détournent aussi notre attention de l'essentiel. La matière nous est plus obéissante, cela nous détourne d'entendre sa parole et son exemple et de comprendre le sens de la nécessité. „238

Sur le plan de l'activité poétique, c'est ce que Henry Bauchau appelle "l'expérience du poème" et cette "exigence de forme sur une matière en partie obscure ${ }^{239}$. En somme, il est, d'un côté, une matérialité obscure de la parole, dont il convient d'être paradoxalement à l'écoute de ce qu'elle suscite, et, d'un autre côté, une forme poétique, qui est une architecture et un cheminement comme Henry Bauchau le laisse entrevoir ${ }^{240}$. C'est exactement ce qui se dit alors qu'Henry Bauchau évoque le 15 août 2004 une lettre de Sylvie Germain à propos de L'Enfant bleu où elle lui écrit: "On pense à Hölderlin, à Artaud, à Van Gogh, à Rothko... à tous les grands visionnaires "bazardifiés" à la folie, à mort, par leurs démons intérieurs. " Et Henry Bauchau lui répond: "Vous parlez à propos de ce livre de Rothko et cela me frappe car mon livre, comme ses tableaux, est fait de couches successives et longuement superposées - non sans obscurité - pour donner le jour à des couleurs nouvelles en allant par les chemins obscurs que sans doute il a connus lui-même."

En somme, à lire attentivement Henry Bauchau, il appert ainsi, d'une part, que la matière est à la fois "parole" et "exempla" et que, d'autre part, nous sommes bien des vivants, mais foncièrement et fondamentalement passifs, puisque nous ne nous sommes certes pas apportés dans la vie; encore que cette passivité peut porter vers un mode d'inattention, qui est un détournement de cette intériorité passive de la vie, voire un oubli de cette condition première, dont on sait désormais qu'elle se déploie avec sa part d'a-temporalité - ou mieux des emboîtements des stases de la temporalité et d'invisibilité. En effet, outre que Simone Weil donne une puissante inflexion à la réflexion sur la matière, dont nous allons mesurer les conséquences quant aux propos sur l'art, elle lui offre aussi une vigoureuse intuition pour penser la temporalité : "Le temps, à proprement parler, n'existe pas et pourtant c'est à cela que nous sommes soumis. Telle est notre condition. Nous sommes soumis à ce qui n'existe pas.” [S. Weil]„„241

238 Nous soulignons.

239 Voir 31 octobre 2002, au sujet du dialogue avec Claude Michel Cluny sur Le Silence de Delphes.

240 Voir ainsi le 30 novembre 2002.

24130 avril 2002. 
Qu'en est-il alors sur le plan de l'expérience personnelle d'Henry Bauchau? En réalité, elle renvoie au plan de l'intériorité, marquée par la marque de "l'inconscient sauvage", qui est dans "dans le mouvement de la vie, ignorant du bien et du mal ${ }^{242}$. À ce propos, une longue citation du Journal mérite d'être faite, en raison de sa valeur illustrative: "L'événement intérieur a eu lieu samedi. En m'éveillant à demi je me suis couché sur le dos. J'ai senti à ce moment que j'étais dans l'être et dans un présent de toujours. Mon passé, mon futur étaient perçus dans ma personnalité propre et non pas niés. Mais j'étais dans l'être du présent, dans un don reçu qui ne s'annonçait pas comme devant durer, mais qui était ce qui est, dans une paix profonde, un non-désir car tout était suffisant, plein, sans failles. J'étais tranquille plus qu'heureux d'un bonheur humain. Tout était solide et pourtant je flottais légèrement. Je savais que j'étais dans mon lit, j'étais dans ce qui est et que je n'avais jamais perçu au milieu de cet immuable apaisement. S'il y avait des couleurs, c'étaient des gris pâles et brillants, de cette couleur de perle, que L., heureuse, m'évoquait toujours. Je percevais autour de moi des présences de marbre, peut-être celles du tombeau. D'un tombeau ouvert et dépassé, comme l'était le temps de la mort. Cet état n'a pas duré longtemps selon nos mesures, peut-être un peu plus d'un quart d'heure mais il était aussi sans limites, il venait d'une grande profondeur de vécu inconscient, car il semble que sans avoir jamais connu rien de semblable je le reconnaissais. /Il ne s'est pas dissipé, j’ai senti qu'il n'était plus là avec une forte injonction à me lever et à me préparer à ma vie de tous les jours. ${ }^{243}$

Je vois encore une autre illustration de cette configuration de la temporalité dans cette importante note du Journal où Henry Bauchau décrit parfaitement l'entremêlée des stases et les puissances de l'invisibilité: "Il y a un mois c'était l'anniversaire de la mort de L. aujourd'hui celui de sa naissance. Depuis sa disparition plus que sa mort, j’ai écrit, mais je n'ai plus vécu, plus vécu comme avant. Il y a deux jours, après la longue contemplation d'une touffe de chardons bleus, j'ai entamé un poème en vers très brefs sur ce que je voyais, ce que je sentais, puis aussi sur ce que je pensais en regardant ces plantes. J'y travaille depuis, j'espère aboutir mais je n'en suis pas sûr. Tant de choses se transforment en écrivant un poème où la forme brève commande, impose son rythme à la langue et à la pensée. / Au point de départ il y a eu le silence. Le silence dans le jardin, le silence des chardons bleus. Des mots ensuite sont venus, des réminiscences. Un vers de Hugo où l'on voit "fleurir le chardon bleu des sables". Souvenirs de mon équipe de jeunes résistants dans le Brabant wallon. Nous étions hébergés dans les granges et le grenier d'un fermier. Pour lui rendre service, nous allions, bien

2423 avril 2002.

24320 avril 2003. Nous soulignons. 
en ligne, couper les têtes des chardons, nombreux à cette époque dans ses vastes champs. ${ }^{244}$

Ainsi, s'il est évident qu'Henry Bauchau pense qu'un "fonds redoutable de sauvagerie" est en l'homme et, singulièrement en lui, comme d'ailleurs le "regret d'une autre Vie plus libre", il estime que la création est une puissance de transformation et de civilisation de ce fonds obscur: "Lart s'adresse toujours en l'homme à une part civilisée, même si cette civilisation est encore primitive aux yeux de celles qui suivent. ${ }^{245} \mathrm{Et}$, voulant faire aussitôt le lien avec le roman s'élaborant, il note à la même date: "La petite fille sauvage est appelée à prendre plus de place dans le roman. Surtout avant le moment où Orion mendie, peut-être est-ce sa présence qui le décide à transmuer ainsi sa violence en appel à l'aide." Il est donc un dédoublement entre le moi et l'histoire, avec un double effet de résonance. Il est aussi une montée des civilisations, mais dans l'intériorité, ce qui amène à nuancer le possible jugement d'un critériologie des civilisations. C'est donc que l'art est bien une expression de la vie et le lieu où l'homme, plus qu'ailleurs, éprouve combien l'approche cognitiviste, intellectualiste, rationaliste est devenue, par l'arrogance de sa quête d'objectivité, radicalement inhumaine.

Le Présent d'incertitude est, à cet égard, d'une extrême richesse. En m'aidant d'une réflexion théorique de Michel Henry, je voudrais ultimement en montrer l'actualisation dans le cas de la création artistique. En effet, Michel Henry fait observer que l'"on peut étendre la démonstration au monde qui lui aussi est fait de formes et de couleurs, et montrer qu'au fond la révélation est double: tout ce qui se montre hors de moi se révèle conjointement comme un développement de la vie en moi. Je crois que c'est cela qui peut rendre au monde non seulement sa poésie, mais aussi cette espèce de chair qu'est l'âme. Et cette chair, c'est ce que nous sommes. L'univers a donc sa réalité dans notre vie en tant qu'elle est dynamique et pathétique "246. N'est-ce pas exactement cela que raconte l'écrivain quand il confie cette expérience pathétique initiée par le regard porté sur un objet singulier: "Je regarde une carte postale, un paysage de Sicile de Nicolas de Staël. Il me semble très mal reproduit et pourtant me touche. Pourquoi? Par les couleurs appliquées, des couleurs vives, le bleu, le rouge, le jaune surtout, qui éclatent sous un ciel brouillé. Ces couleurs raniment en moi des plaisirs, des sensations, des instants évanouis. Surtout elles font vivre, en dehors de toute réalité, l'espérance d'un monde plus chaud, plus éclatant que celui que j'ai connu. Il y a en nous le désir d'une intensité qui n'est pas dans les surfaces que nous pouvons voir et qui n'est sans doute que dans

24418 juillet 2004 .

24530 juillet 2002 .

246 Michel Henry, Entretiens, Arles, Sulliver, 2005, p. 110. Nous soulignons. 
les visions intérieures où nous les inventons par instants. Nicolas de Staël, comme Gauguin, a connu ces visions que je tente de susciter aussi avec des mots. " 247 ?

C'est donc bien la notion de "pathos-avec" qui est au centre du dispositif et forme la "communauté pathétique", avec les forces et les affects qui lui sont propres, telles, par exemple, ces tonalités de la peinture de Staël, qui n'est donc pas seulement "la figuration de choses extérieures mais l'expression de leur réalité intérieure ${ }^{248}$. Il est donc infiniment plus que la visibilité de cette carte postale défraîchie, il est une vie spécifique invisible de chaque couleur qui est une impression charnelle, intérieure et émotionnelle, capable de faire sentir le dynamique de la vie. Or s'il en est ainsi, c'est que la création artistique instaure bien une sorte de communauté pathétique où, dans ce cadre, les couleurs, comme d'ailleurs les formes, sont aussi des forces ayant une réalité émotive et sentie.

Aussi, ayant déjà évoqué l'apport théorique de Michel Henry, on pourra encore rappeler, en guise de confirmation de la posture d'Henry Bauchau, que "la théorie des formes, qui renvoie à des forces, renvoie du même coup à la subjectivité, parce que les forces habitent notre corps, notre corps vécu, notre corps subjectif qui est notre corps réel. Par conséquent le monde des formes est, en quelque sorte, un univers chiffré dont la vraie signification renvoie au jeu des forces en nous, donc à la vie, car le corps vivant est un corps qui est fait de forces: telle est l'origine de la peinture. Ici encore, c'est un élément invisible, la force invisible avec laquelle s'identifie le corps vivant, qui est le principe de la composition de la peinture „249.

C'est en ce sens que l'art peut exprimer la vie - dont on rappellera bien qu'ils ne sont pas similaires -, mais aussi l'affectivité, tout en renvoyant au corps vivant. Comme c'est également autour des formes et des couleurs, entendues comme des tonalités, que s'éprouve une communauté du voir et du sentir, même si c'est sur un mode imaginaire, car les formes sont le lieu du recoupement entre les forces des corps vivants, qu'il s'agisse du créateur ou du spectateur ${ }^{250}$. La création artistique offre ainsi de vivre, en quelque

\footnotetext{
24731 janvier 2003.

248 Michel Henry, "Pour une phénoménologie de la communauté", dans Phénoménologie matérielle, Paris, PUF, 1990, p. 179.

249 Michel Henry, "Art et phénoménologie de la vie", dans Phénoménologie de la vie. Tome III. De l'art et du politique, op. cit., p. 292.

250 Michel Henry note encore que "l'intersubjectivité s'accomplit dans la mesure où le tableau est un ensemble, non pas de formes mais de forces, non pas de couleurs extérieures transcendantes, mais d'impressions et d'émotions. À ce moment-là, il y a contemporanéité: le spectateur se fait le contemporain des forces et des impressions que recrée en lui le tableau comme imaginaire, dans son apparence extérieure. [...] Être contemporain, cela veut dire répéter dans une répétition intérieure, dans la réactualisation de ce qui avait été actualisé autrefois. [...] Dans le cadre de la peinture, la contemporanéité c'est cette texture de forces et d'émotions intérieures dont le tableau est l'expression." Dans Michel
} 
sorte, des expériences qui pourraient être appelées "pures", parce que précisément pathétiques, en somme des expériences qui sont de l'ordre d'une reviviscence affective, comme celle du regard porté sur les couleurs d'une peinture, capable de laisser advenir une communauté esthétique génératrice de contemporanéité, mais surtout une inscription dans l'indéchiffrable et une visée dans l'espérable.

De ce point de vue, c'est incontestablement la puissance de l'immanence que, j'en fais l'hypothèse, Henry Bauchau tente de penser et d'écrire, de vivre bien sûr, dans la mesure où l'art apparaît comme un singulier pouvoir de modification du trauma, ce pouvoir d'autotransformation, mais selon une modification qui n'est pas de l'ordre d'une représentation (ou d'une prise de conscience) car c'est d'une épreuve et d'un approfondissement précisément immanents dont il est question. Henry Bauchau est explicite quand il évoque le rapport entre l'art et ses puissances vitales: "Je retrouve dans le Journal d'Antigone une pensée de Georges Braque qui n'a cessé depuis de m'interroger: "Il faut descendre jusqu'au chaos primordial et s'y sentir chez soi." Il me semble que cette phrase serait bien à sa place en exergue de mon roman en travail. C'est tout l'effort de Véronique pour aider Orion à se sentir un peu "chez soi" dans le monde et dans l'art. Ponge éclaire bien la pensée de Braque en remarquant que "le chaos, mot grec, signifiait paradoxalement à l'origine: ouverture et abîme, c'est-à-dire libération, non pas de la maladie, mais d'une part suffisante de nos forces pour qu'on puisse faire œuvre, œuvre d'art et survivre”." „51

Et c'est précisément sur ce point qu'Henry Bauchau montre avec finesse comment cet acte de vie intérieure ou de sur-vivre se déploie le plus souvent à partir d'une matière, dont on sait combien, pour l'écrivain inspiré, elle parle un langage spécifique, empreint de potentialités spécifiques. Ainsi, Henry Bauchau note: "Brancusi: "Faire n'est rien, il faut se mettre en état de faire." C'est par une longue évolution psychologique, par l'amitié et l'imagination du travail que Lionel s'est "mis en état de faire". Peintre, graveur, sculpteur il vit dans une remarquable proximité des matières qu'il emploie, dans l'amour du bois qui ne connaît pas l'angoisse et s'éclaire de patience. ${ }^{252}$ C'est de la sorte que l'art trouve certes, d'une part sa fonction thérapeutique, mais, d'autre part, est également ce lieu spécifique et matériel dont les limites qu'il laisse advenir sont la source d'un possible salut. Henry Bauchau confie ainsi : "Lartiste obsessionnel, comme il y en a beaucoup, se défend peut-être par ses obsessions contre des états qu'il vit sans en être pleinement conscient et qui risqueraient d'être pris pour de la

Henry, "Art et phénoménologie de la vie", dans Phénoménologie de la vie. Tome III. De l'art et du politique, op. cit., p. 294.

25118 juillet 2003 .

25228 septembre 2005 . 
folie par la société de son temps. Le choix de l'art qu'on sert est peut-être commandé de même par l'enflure de l'ego qu'une autre forme plus difficile risquerait de provoquer. Si j'avais réussi, comme je l'ai tant espéré, comme poète dramaturge, ce succès me serait sans doute monté à la tête, si une autre ouvre peut-être plus riche s'était ainsi développée cela aurait été au détriment de mon être. Ainsi beaucoup de gens pensent que l'inflation de la société ou l'inflation du moi favorise la créativité et l'action, mais il y a aussi un danger d'emballement, de dépassement dionysiaque. [...] La sculpture par ses limitations a certainement aidé Lionel à ne pas sortir, dans sa période analytique, des limites du tolérable et d'être considéré comme un artiste et pas seulement comme un malade. ${ }^{253}$

On le voit, pour Henry Bauchau, "l'art est une voie rude et risquée, mais on y vit de façon ardente et dans une jeunesse des perceptions et sentiments que je n'ai plus, quant à moi, dans la vie courante ${ }^{254}$. Ceci revient en tout cas à dire que si tout savoir du monde, comme lieu de la technique ou du dionysiaque, doit être relativisé, il ne peut pour autant présupposer la disparition du savoir de la Vie, ce savoir plus essentiel, souvent invisible; en tout cas un savoir qui redonne au corps, mieux à la chair, toute ses virtualités et potentialités, surtout quand elles passent au travers des expressions artistiques par la médiation de la puissance énergétique de la matière. En somme, pour croiser ici les expériences d'Henry Bauchau avec l'apport théorique de Michel Henry, on s'accordera à faire valoir, comme le pense le philosophe, que "l'art veut nous faire voir, au-delà de la chose, l'apparaître qui se cache et dans lequel la chose se dévoile, mais qu'elle cache en même temps: cette sorte de faire-valoir qui est caché „255.

C'est sans doute en ce sens qu'il est possible de comprendre ce vou d'Henry Bauchau, alors qu'il clôture son Journal: "Peut-être y aura-t-il un jour un retour vers des formes plus simples, où le texte, les corps et les voix des comédiens seront présents seuls. Il est possible que le théâtre et les arts, dans la sophistication de tout, redeviennent le lieu des moyens simples, du sens et de la vérité corporelle. Ce ne sera pas tout de suite ni pour toujours. ${ }^{256}$

Jean Leclercq

Université catholique de Louvain

25319 décembre 2005.

25416 mai 2003.

255 Michel Henry, "Art et phénoménologie de la vie", dans Phénoménologie de la vie. Tome III. De l'art et du politique, op. cit., p. 284.

25620 décembre 2005. 